\title{
Efecto de aplicaciones de calcio en la calidad de la fruta de arándano alto (Vaccinium corymbosum L.) cv. Elliot
}

\author{
Effect of calcium applications on the quality of highbush blueberry fruit cv. Elliott
}

\author{
Tomás Lobos Manríquez ${ }^{1}$, Hernán Pinilla Quezada², Walter Lobos Alvarez ${ }^{3}$
}

\begin{abstract}
RESUMEN
La producción comercial de arándanos en el país ha sufrido modificaciones que apuntan hacia garantizar el cumplimiento de las normas de calidad impuestas por los países importadores. Con el objetivo de evaluar el efecto de distintas fuentes de calcio sobre la calidad de frutos de arándano alto, en la temporada agrícola 2007/2008, se realizaron aplicaciones de calcio al suelo y foliar en precosecha en plantas de arándano alto (Vaccinium corymbosum L.) cultivar Elliott. El estudio se realizó en un huerto comercial localizado en la comuna de Río Negro, Región de Los Lagos. El suelo correspondió a un andisol de la serie Corte Alto. El ensayo consideró siete tratamientos y las aplicaciones se realizaron en plantas de 15 años de edad, desde inicio de cuaja hasta desarrollo de frutos. Se llevaron a cabo diez cosechas donde se evaluaron calibre, peso, sólidos solubles y firmeza de frutos de arándano alto. Los datos obtenidos se sometieron a un análisis de varianza (ANOVA) y prueba de comparación múltiple mediante la prueba de Tukey con un nivel de significancia del $5 \%$. No se produjeron diferencias estadísticamente significativas entre los diferentes tratamientos evaluados para las variables de calibre, peso y sólidos solubles. Aplicaciones de calcio foliar y al suelo en precosecha, desde cuaja hasta desarrollo de frutos, ocasionaron diferencias significativas en la firmeza de éstos, respecto de las plantas no tratadas.

Palabras clave: Calidad de frutos, Vaccinium corymbosum L., aplicaciones de calcio.
\end{abstract}

\begin{abstract}
The commercial production of highbush blueberries (Vaccinium corymbosum L.) in Chile has undergone modifications that aim towards ensuring compliance of quality standards imposed by the importing countries. In order to evaluate the effects of different sources of calcium on highbush blueberry quality, in the agricultural season of 2007/2008 foliar and soil applications of calcium were applied to Elliott blueberry plants. The trial was performed in a commercial orchard located in the commune of Rio Negro, Los Lagos region. Soil was an andisol corresponding to the Corte Alto series. The experiment occupied seven treatments; the applications were made on 15 year old plants, from blooming until fruit development. Ten harvests were carried out to evaluate size, weight, soluble solids and firmness of highbush blueberry fruit. Treatment means were compared by an analysis of variance (ANOVA) and Tukey multiple range test at the 5\% level when significant differences were found. No statistically significant differences were found between the different treatments evaluated for the variables of size, weight and soluble solids. Fruit firmness was the only evaluated factor that showed statistically significant differences with respect to untreated plants.
\end{abstract}

Key words: Fruit quality, Vaccinium corymbosum L., calcium applications.

\section{Introducción}

La superficie plantada con arándano alto (Vaccinium corymbosum L.) en el país durante los últimos años ha producido un aumento importante de la oferta exportable y esto ha influido en el aumento de los estándares de calidad requeridos por los países importadores.
La deficiencia de calcio en los frutos es un problema que se asocia más a una deficiente translocación de este elemento por los vasos conductores floemáticos de la planta, que a la cantidad total de calcio presente en el suelo (Faust, citado por Aedo, 1994).

Estudios realizados por Arredondo (1994), Conway et al. (1994), Flores (2004) y Nappier y

\footnotetext{
1 Ingeniero Agrónomo. HYPERLINK “mailto:tlobos@gmail.com” tlobos@gmail.com

2 Ingeniero Agrónomo, Magíster en Fertilidad de Suelos. Académico Universidad de La Frontera. HYPERLINK “mailto:hpin@ ufro.cl”hpin@ufro.cl

3 Ingeniero Agrónomo. Académico Universidad de La Frontera. HYPERLINK “mailto:wlobos@ufro.cl” wlobos@ufro.cl
}

Fecha de Recepción: 09 Septiembre, 2010.

Fecha de Aceptación: 11 Febrero, 2011. 
Combrink (2006) indican que con la disminución de la concentración de calcio habría un aumento en la susceptibilidad de la fruta al ataque fungoso en poscosecha y menor firmeza del fruto (Hanson, 1995b).

Normalmente el suministro de nutrientes en frutales es al suelo, logrando de esta manera satisfacer los requerimientos de las plantas para su crecimiento vegetativo y producción de la fruta. Sin embargo, desórdenes fisiológicos inducidos por niveles deficitarios de calcio en los frutos han hecho frecuentes las aspersiones de productos a base de calcio desde cuaja hasta desarrollo de frutos (Arredondo, 1994; Aedo, 1994; Flores, 2004; Rubilar, 2004; Stückrath et al., 2008) y las inmersiones en soluciones de sales de calcio en poscosecha (Hanson et al., 1993).

Las aplicaciones de calcio al suelo se recomienda realizarlas temprano en la temporada, entre julio o agosto, para que tengan incidencia en el proceso productivo (Korcak, 1988). La fuente de calcio a utilizar es de suma importancia para incidir en los aspectos de calidad de los frutos (Hanson y Berkheimer, 2004; Rubilar, 2004).

El presente estudio tiene por objetivo determinar el efecto del aporte de calcio foliar y al suelo en la calidad de frutos de arándano alto (Vaccinium corymbosum L.) cv. Elliott.

\section{Materiales y Métodos}

El estudio se realizó durante la temporada de cosecha 2007-2008, en el predio de la empresa "Agrícola Trucao" (4049' 41,18" de latitud Sur y $73^{\circ} 11$ ' 45,27" de longitud Oeste), distante a 4 kilómetros al sur de la localidad de Río Negro, comuna de Río Negro, Región de Los Lagos, Chile. El suelo del huerto pertenece a la serie Corte Alto, posee una topografía ondulada con pendientes complejas entre 2 a $5 \%$ y un drenaje bueno (Tosso, 1985). La zona donde se realizó el ensayo posee un régimen pluviométrico anual que fluctúa entre los $1.200 \mathrm{y}$ $1.500 \mathrm{~mm}$; otoño $400-500 \mathrm{~mm}$; invierno $700 \mathrm{~mm}$; primavera $250-400 \mathrm{~mm}$ y verano $150-200 \mathrm{~mm}$.

La caracterización química del suelo utilizado en el estudio se indica en el cuadro 1.

La especie utilizada fue arándano alto (Vaccinium corymbosum L.) cultivar "Elliott", que se caracteriza por presentar plantas muy productivas, con hábito de crecimiento erecto, alto rendimiento y rusticidad. El fruto es de tamaño mediano, de color muy
Cuadro 1. Análisis de suelo del sitio del ensayo a 0-20 cm. Chemical analysis from assay soil 0-20 cm depth.

\begin{tabular}{lcc}
\hline Análisis & Unidad & Valor \\
\hline pH en agua & Rel. 1:2.5 & 5,89 \\
Mat. Orgánica & $\%$ & 19,8 \\
Fósforo Olsen & $(\mathrm{mg} / \mathrm{kg})$ & 29,8 \\
Nitrógeno-nitrato & $(\mathrm{mg} / \mathrm{kg})$ & 48,1 \\
Potasio de intercambio & $\left(\mathrm{cmol}^{+} / \mathrm{kg}\right)$ & 1,19 \\
Calcio de intercambio & $\left(\mathrm{cmol}^{+} / \mathrm{kg}\right)$ & 8,58 \\
Magnesio de intercambio & $\left(\mathrm{cmol}^{+} / \mathrm{kg}\right)$ & 3,77 \\
Sodio de intercambio & $\left(\mathrm{cmol}^{+} / \mathrm{kg}\right)$ & 0,23 \\
Aluminio de intercambio & $\left(\mathrm{cmol}^{+} / \mathrm{kg}\right)$ & 0,09 \\
Aluminio extractable & $(\mathrm{mg} / \mathrm{kg})$ & 616 \\
Suma de bases & $(\mathrm{cmol} / \mathrm{kg})$ & 13,8 \\
Saturación de aluminio & $\%$ & 0,68 \\
Azufre & $(\mathrm{mg} / \mathrm{kg})$ & 27,6 \\
Fierro & $(\mathrm{mg} / \mathrm{kg})$ & 186 \\
Manganeso & $(\mathrm{mg} / \mathrm{kg})$ & 55,9 \\
Zinc & $(\mathrm{mg} / \mathrm{kg})$ & 7,86 \\
Cobre & $(\mathrm{mg} / \mathrm{kg})$ & 32 \\
Boro & $(\mathrm{mg} / \mathrm{kg})$ & 1,87 \\
\hline
\end{tabular}

azul en estado maduro, con una cicatriz pequeña. Es resistente a Monilia sp. y estudios en Chile han determinado que la fruta presenta un alto nivel de acidez. Es una variedad de maduración tardía, presentando una época de cosecha en la zona del ensayo que va desde fines de enero a fines de marzo (Godoy, 2002).

Los tratamientos definidos para la presente investigación fueron los siguientes (Cuadro 2).

Para este estudio se seleccionaron plantas sanas y homogéneas, según sus características de tamaño y vigor. El manejo agronómico fue igual en todo el huerto; éste considera un control fitosanitario, con los estándares de calidad que se exigen para la exportación de esta fruta, al igual que la fertilización y riego que se aplicaron según sus requerimientos y por lo tanto todos ellos pasaron a ser no influyentes en los resultados del estudio. La fertilización del huerto se realizó con $100 \mathrm{~kg}$ de N/ha, $70 \mathrm{~kg}$ de $\mathrm{P}_{2} \mathrm{O}_{5} /$ ha y $100 \mathrm{~kg}$ de $\mathrm{K}_{2} \mathrm{O}$.

El volumen de agua utilizado para las aplicaciones de calcio fue de 600 litros por hectárea, aplicados mediante una bomba de espalda marca SOLO de 15 litros. La dosis utilizada en cada tratamiento como aspersión correspondió a la dosis recomendada por las empresas que comercializan los productos.

La aplicación de calcio se realizó 12 días después de plena flor, con frutos de cinco mm de diámetro como promedio. Este estado se observó el día 24 
de noviembre. Se realizaron tres aplicaciones con un intervalo de ocho días entre cada aplicación.

El diseño experimental fue de bloques al azar con tres repeticiones por tratamiento. La unidad experimental fue una parcela constituida por cuatro plantas.

Se realizaron diez cosechas recolectando manualmente la fruta en color de cosecha, entre las 8:00 a 11:00 hrs. La evolución de madurez de cosecha de los frutos del ensayo fue llevada a cabo según metodología de evaluación de madurez propuesta por Michigan Blueberry Growers (MBG, 1998).

Después de la recolección de la fruta en el huerto, ésta fue llevada al packing donde permaneció una hora a $12{ }^{\circ} \mathrm{C}$ para disminuirle la temperatura. Posteriormente se procedió a elegir al azar una muestra de 50 frutos de cada repetición por cada tratamiento, con el propósito de evaluar calibre, peso, sólidos solubles y firmeza.

Los datos obtenidos se sometieron a un análisis de varianza (ANOVA) con un nivel de significancia del 5\%. Cuando los datos analizados registraron diferencias significativas entre los tratamientos, se aplicó prueba de comparación múltiple de Tukey $(\mathrm{p} \leq 0,05)$. Se utilizó el software SPSS 11.5 para la evaluación estadística.

\section{Resultados y Discusión}

\section{Calibre de frutos}

En la Figura 1 se presentan los resultados obtenidos de calibre del fruto para cada uno de los tratamientos evaluados. Se observan calibres muy similares entre los tratamientos evaluados, con rangos de valores entre 13,8 y 14,2 $\mathrm{mm}$. No se produjeron diferencias significativas de los tratamientos en el calibre. Estos resultados serían similares a los obtenidos por Koron et al. (2009) quienes no encontraron variación en el tamaño de frutos de arándano alto con aspersiones de calcio respecto del tratamiento control. Poovaiah et al. (1988) y Yuri (1995) señalan que, sobre la base del rol del calcio en la planta, los resultados obtenidos en este ensayo estarían dentro de lo esperado, donde el calcio juega sólo un papel indirecto en el tamaño de los frutos.

\section{Peso de frutos}

En la Figura 2 se presenta el peso del fruto para cada tratamiento evaluado. El peso del fruto estuvo en el rango comprendido entre 1,37 y 1,54 gramos, sin encontrarse diferencia significativa en el peso de éstos por efecto de los tratamientos evaluados. Los resultados obtenidos en este ensayo coinciden con la experiencia realizada por Koron et al. (2009), quienes al realizar aplicaciones foliares de calcio en plantas del cultivar Bluecrop no encontraron diferencias significativas en el peso de los frutos manejados con calcio y el tratamiento testigo.

\section{Sólidos solubles}

De acuerdo a los resultados obtenidos para los sólidos solubles (Figura 3), no se produjeron diferencias significativas entre los tratamientos. Eck (1988) cita el rango comprendido entre 12 a $15^{\circ}$ Brix como los valores frecuentes de sólidos solubles presentes en arándano alto. Los valores obtenidos en esta investigación estuvieron entre 12,2 y $12,8^{\circ}$ Brix durante la época de cosecha.

Los datos presentados concuerdan con los obtenidos por Flores (2004) y Koron et al. (2009), quienes no encontraron diferencias significativas en el contenido de sólidos solubles al realizar aspersiones foliares de calcio y compararlas con el tratamiento sin aplicación de este nutriente.

Cuadro 2. Tratamientos, productos y dosis aplicado en arándano alto cv. Elliott.

Treatments, products and applied doses in highbush blueberry cv. Elliott.

\begin{tabular}{llcc}
\hline Tratamiento & Producto & Cantidad de calcio/aplicación/ha & Dosis producto/ha \\
\hline T-1 & Testigo sin aplicaciones & - & - \\
T-2 & Nitrato de calcio & $31 \mathrm{~kg}$ & $166 \mathrm{~kg}$ \\
T-3 & Carboxy calcio & $160 \mathrm{~g}$ & $2 \mathrm{~L}$ \\
T-4 & Defender calcio & $378 \mathrm{~g}$ & $2 \mathrm{~L}$ \\
T-5 & Borocal & $143 \mathrm{~g}$ & $2 \mathrm{~L}$ \\
T-6 & Foli-cal & $143 \mathrm{~g}$ & $2 \mathrm{~L}$ \\
T-7 & Metalosate calcio & $366 \mathrm{~g}$ & $2 \mathrm{~kg}$ \\
\hline
\end{tabular}




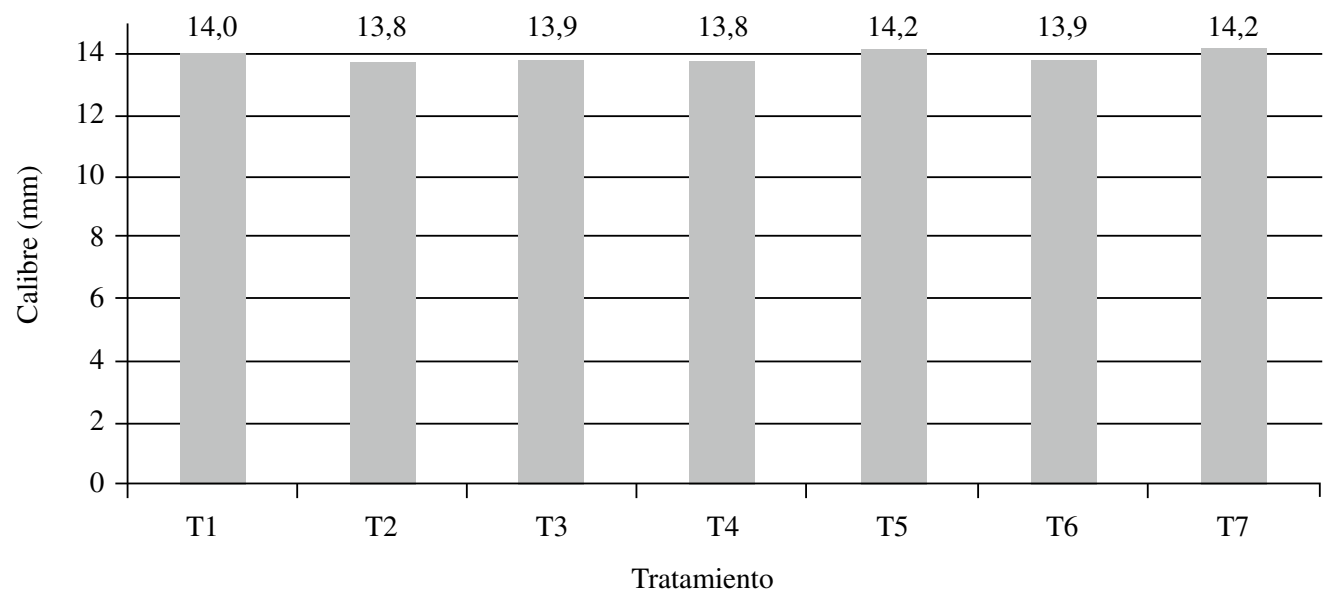

Figura 1. Efecto de los tratamientos en el calibre de frutos de arándano alto cv. Elliott. Valores expresados en milímetros. Effect of treatments in the size of highbush blueberry fruit cv. Elliott. Values expressed in millimeters.

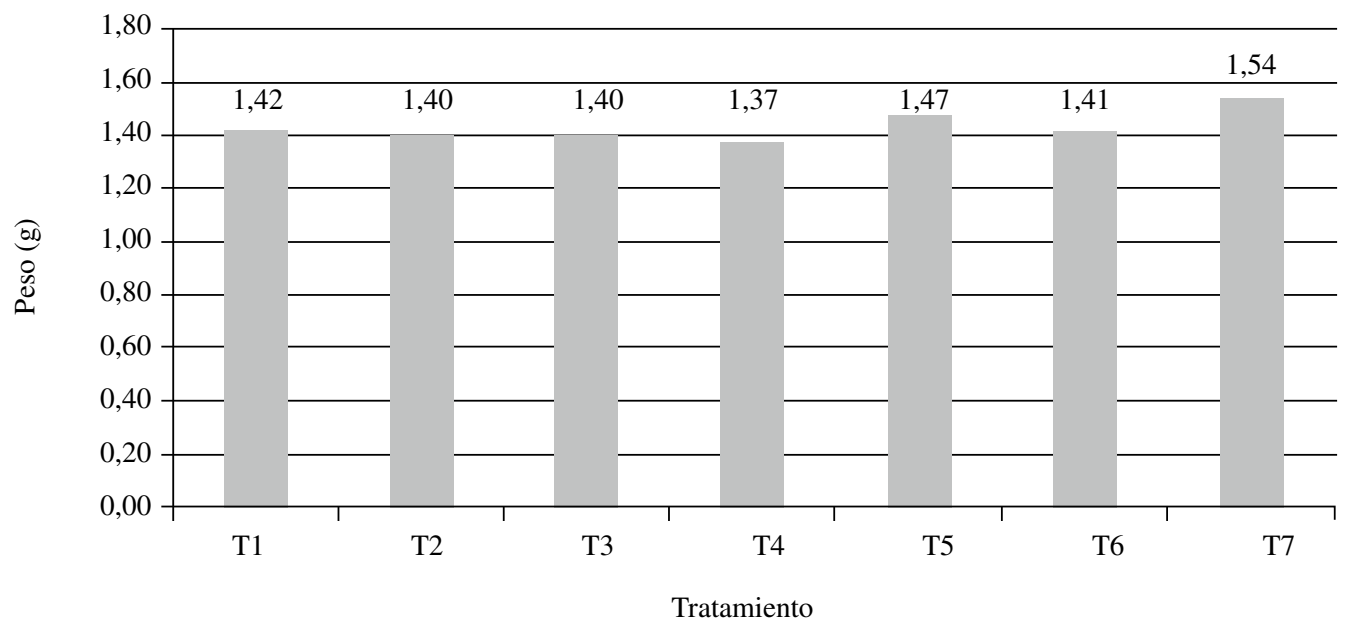

Figura 2. Efecto de efecto de los tratamientos en el peso por fruto de arándano alto cv. Elliott. Valores expresados en gramos. Effect of treatments in the weight of highbush blueberry fruit $c v$. Elliott. Values expressed in grams.

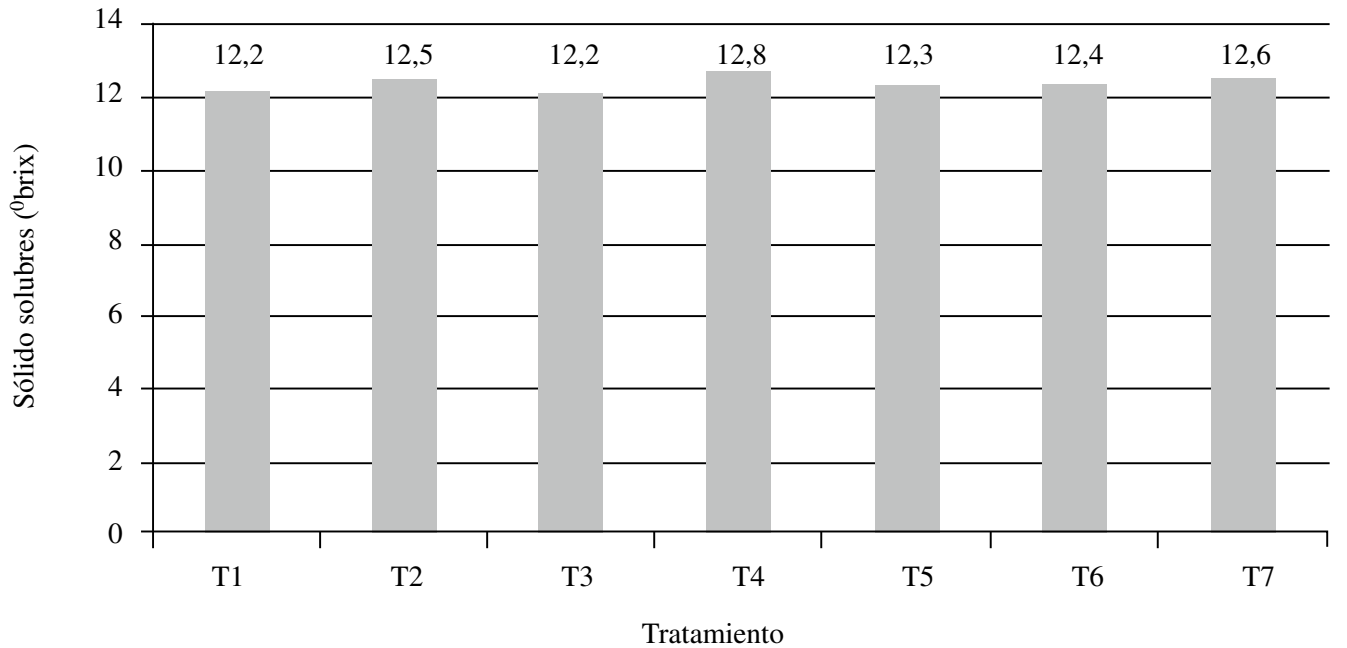

Figura 3. Efecto de los tratamientos en el contenido de sólidos solubles de frutos de arándano alto cv. Elliott. Valores expresados en grados Brix.

Effect of treatments in the total soluble solids of highbush blueberry fruit cv. Elliott. Values expressed in Brix. 


\section{Firmeza de los frutos}

La Figura 4 muestra los valores correspondientes a firmeza de los frutos de cada uno de los tratamientos del ensayo. El rango de valores estuvo entre 0,66 y 0,72 libras/pulgada ${ }^{2}$. Los resultados muestran que hubo diferencias significativas entre los tratamientos al medir la firmeza a la cosecha. Estos resultados guardan relación con la respuesta obtenida por Aedo (1994), Flores (2004) y Rubilar (2004), todos los cuales señalan que aplicaciones de calcio en precosecha mejoran la firmeza de la fruta. Hanson et al. (1993) también encontraron mayor firmeza en frutos de arándano alto al sumergirlos en soluciones de calcio, pero en poscosecha.

El tratamiento testigo (T1) que no recibió aplicaciones de calcio fue el tratamiento que obtuvo la firmeza más baja. El resto de los tratamientos tuvo un comportamiento similar, destacándose positivamente la mayor firmeza obtenida por el tratamiento con Defender calcio. El resto de las aplicaciones con productos foliares tuvo similar resultado con el tratamiento con nitrato de calcio al suelo. Al respecto, Bramlage (1995) postula que aplicaciones de nitrato de calcio son efectivas y posiblemente menos tóxicas que otros productos, pero deben utilizarse dosis más altas, ya que por ser aplicadas al suelo interaccionan con otros cationes presentes en el complejo de intercambio. Ruiz
(1995) señala además que el calcio es fácilmente lixiviable en suelos ácidos, lo que hace necesario un mayor número de aplicaciones para mejorar la absorción de este elemento y así equiparar el efecto producido por aplicaciones foliares.

Hanson (1995a) no encontró un aumento en la firmeza de frutos de arándano alto cultivar Bluecrop, al asperjar cloruro de calcio. Estas diferencias encontradas podrían explicarse, por ejemplo, por el manejo de las plantas, medio ambiente, diferencias varietales y distintas formulaciones y dosis de los productos utilizados en cada experimento.

\section{Conclusiones}

Sobre la base de los resultados obtenidos en este trabajo se puede concluir que:

- No hubo diferencia estadísticamente significativa entre los diferentes tratamientos evaluados para las variables de calibre, peso y sólidos solubles.

- Se obtuvieron diferencias estadísticamente significativas con las aplicaciones de calcio en precosecha, desde cuaja hasta desarrollo de frutos, para la variable firmeza, respecto de las plantas no tratadas.

- La aplicación de calcio al suelo y por vía foliar no presentó diferencias significativas para las variables evaluadas. Por lo tanto, la aplicación de calcio al suelo es una alternativa promisoria por su menor costo.

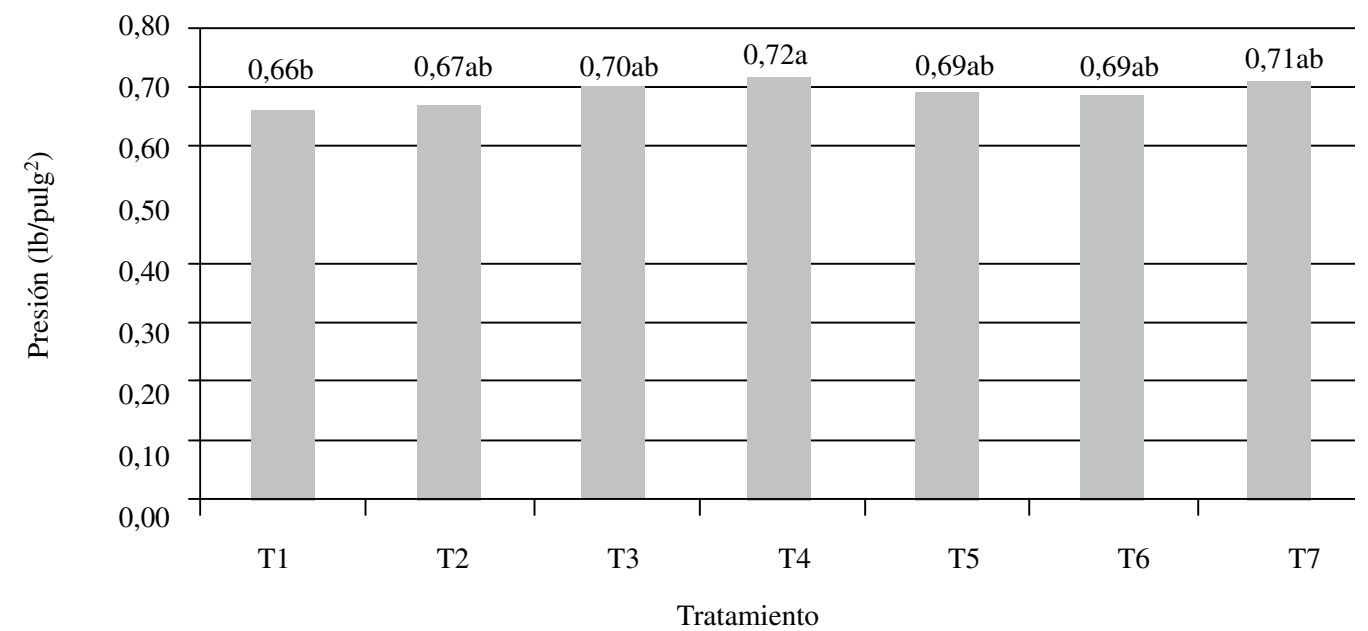

Figura 4. Efecto de los tratamientos en la firmeza de frutos de arándano alto cv. Elliott. Valores expresados en libras/pulgada ${ }^{2}$. Effect of treatments in the firmness of highbush blueberry fruit cv. Elliott. Values expressed in lb/inch ${ }^{2}$. 


\section{Literatura Citada}

Aedo, $\mathrm{P}$.

1994 Efecto de aspersiones de sales de calcio en precosecha sobre frutos de arándano alto c.v. Bluecrop. Tesis Ing. Agrónomo. Universidad de La Frontera. Temuco. 83 p.

Arredondo, G.

1994 Calcio en arándanos: gradiente natural en el fruto y respuesta a aplicaciones en precosecha. Tesis Ing. Agrónomo. Universidad de Talca. Talca. $46 \mathrm{p}$.

Bramlage, W.

1995 Calcio y desórdenes fisiológicos. Symposium internacional: Calcio en fruticultura. Universidad de Talca. Talca, Chile, pp. 73-81

Conway, W.S.; Sams, C.E. y Kelman, A.

1994 Enhancing the natural resistance of plant tissues to postharvest diseases through calcium applications. Hortscience. 29: 751-754.

Eck, P.

1988 Blueberry Science. Rutgers Univ. Press, New Brunswick, N.Y, pp. 200-212.

Flores, A.

2004 Efecto de aspersiones de tres productos a base de calcio en precosecha sobre la calidad postcosecha de frutos de arándano c.v. Berkeley. Tesis Ing. Agrónomo. Universidad de Chile. Santiago. 26 p.

Godoy, O.

2002 Incidencia del prefrío en la calidad de fruta de arándano alto (Vaccinium corymbosum L.) cv. Elliott, según estado de madurez de cosecha y período de almacenaje convencional. Tesis Ingeniero Agrónomo. Universidad de La Frontera. Temuco, Chile. 69 p.

Hanson, E.; Beggs, J. y Beaudry, R.

1993 Applying calcium chloride postharvest to improve highbush blueberry firmness. HortScience 28(10): 1033-1034.

Hanson, E.

1995a Preharvest calcium sprays do not improve highbush blueberry (Vaccinium corymbosum L.) quality. HortScience 30(5): 177-179.

Hanson, E.

1995b Calcio en berries y vides. Symposium internacional: Calcio en fruticultura. Universidad de Talca. Talca, Chile, pp. 142-149.
Hanson, E. y Berkheimer, S

2004 Effect of Soil Calcium Applications on Blueberry Yield and Quality. Available at http://www.haworthpress.com/ store/ArticleAbstract.

Korcak, R.F.

1988 Nutrition of blueberry and other calcifuges. Horticultural Reviews. 10: 183-227.

Koron, D.; Sturm, K. y Pavlin, S.

2009 Effects of Ca foliar fertilizers on fruit quality of highbush blueberry. Acta Horticulturae 810: 705-708

Michigan Blueberry Growers Marketing.

1998 Blueberry Granding. O.O. Drawer B, Grand Juction, MI 49056.

Nappier, D.R. y Combrink, N.J.J.

2006 Aspects of Calcium Nutrition to Limit Plant Physiological Disorders. Acta Horticulturae 702: 107-113.

Poovaiah, B.; Glenn, G. y Reddy, A

1988 Calcium and fruit softening: physiology and biochemistry. Horticultural Reviews. 10: 107-143.

Rubilar, E.A.

2004 Evaluación del efecto de la aplicación de calcio en frutos de arándano alto (Vaccinium corymbosum L. cv. Patriot). Tesis Ing. Agrónomo. Universidad de Concepción. 24 p.

Ruiz, R.

1995 Calcio: disponibilidad en el suelo y su absorción por la planta. Symposium internacional: Calcio en fruticultura. Universidad de Talca. Talca, Chile, pp. 1-24.

Stückrath, R.; Quevedo, R.; Fuente, L. de la; Hernández, A. y Sepúlveda, V.

2008 Effect of foliar application of calcium on the quality of blueberry fruits. Journal of Plant Nutrition 31(7): 1299-1312

Tosso, J.

1985 Suelos Volcánicos de Chile. Instituto de Investigaciones Agropecuarias, p. 617.

Yuri, J.

1995 Aspectos fundamentales de la bioquímica y fisiología del calcio. Symposium internacional: Calcio en fruticultura. Universidad de Talca. Talca, Chile, pp. 25-36. 